\title{
Ciencia e innovación tecnológica en la salud en Cuba: resultados en problemas seleccionados
}

\author{
Nereida Rojo Pérez, ${ }^{1}$ Carmen Valenti Pérez, ${ }^{1}$ Nelcy Martínez Trujillo, ${ }^{1}$ \\ Ileana Morales Suárez, ${ }^{2}$ Eric Martínez Torres, ${ }^{3}$ Ileana Fleitas Estévez, ${ }^{4}$ \\ Miriam Portuondo Sao, ${ }^{2}$ Yisel Torres Rojo ${ }^{3}$ y V. Gustavo Sierra González ${ }^{5}$
}

Forma de citar: $\quad$ Rojo Pérez N, Valenti Pérez C, Martínez Trujillo N, Morales Suárez I, Martínez Torres E, Fleitas Estévez I, et al. Ciencia e innovación tecnológica en la salud en Cuba: resultados en problemas seleccionados. Rev Panam Salud Publica. 2018;42:e32. https://doi.org/10.26633/RPSP.2018.32

RESUMEN En Cuba, la investigación para la salud se basa en las prioridades de la política científica nacional, derivadas del estado de salud de la población. El objetivo de este artículo es describir las características del Sistema de Ciencia e Innovación Tecnológica en el sector y cómo los resultados de sus investigaciones benefician la salud de los grupos poblacionales. Para ello se seleccionaron investigaciones relacionadas con la generación de productos y tecnologías, la diabetes, el dengue y la discapacidad. Este sistema sigue los preceptos metodológicos del Ministerio de Ciencia, Tecnología y Medio Ambiente y cuenta con 37 entidades de investigación. Se organiza en programas y proyectos que favorecen la investigación básica y aplicada, con un enfoque multidisciplinario e intersectorial; estos son financiados mayormente por el Estado y organizados en ciclos cerrados o completos, es decir, una misma entidad se encarga de todo el proceso, desde la investigación hasta la comercialización, incluidos los estudios de mercados y la vigilancia poscomercialización. Las investigaciones seleccionadas evidencian la armonía entre la investigación, la generalización de los resultados y su efecto en mejorar la salud y el acceso universal de la población. Se lograron resultados en métodos de diagnóstico, vacunas preventivas y terapéuticas, signos de alarma para el pronóstico y tratamiento del dengue, prevención de malformaciones congénitas, y políticas y programas que han beneficiado a las personas con discapacidad y sus familiares. La voluntad del Estado para desarrollar y financiar la investigación científica, la acción intersectorial, la definición de las prioridades de investigación, y la preparación y atención sistemática del capital humano han sido factores determinantes para el cumplimiento de los objetivos del sistema.

Palabras clave Política nacional de ciencia, tecnología e innovación; gestión de ciencia, tecnología e innovación en salud; investigación biomédica; planes y programas de salud; proyectos de investigación; Cuba.

El Sistema de Ciencia e Innovación Tecnológica en Cuba está regido por el Ministerio de Ciencia, Tecnología y Medio Ambiente (CITMA), de acuerdo con

\footnotetext{
Escuela Nacional de Salud Pública, La Habana, Cuba. Enviar la correspondencia a Nereida Rojo Pérez, nereida.rojo@infomed. sld.cu
}

la Resolución No. 44/2012, cuya implementación consolidó la reorganización de la actividad científica a partir de una política dirigida a lograr más eficiencia y vinculación con la actividad productiva y de servicios (1). Esto significó una reducción del número de programas de investigación y de las estructuras, y el

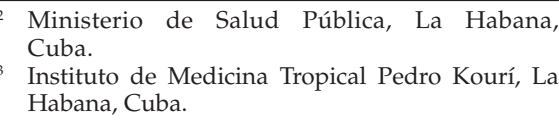

Ministerio de Salud Pública, La Habana, Cuba.

Instituto de Medicina Tropical Pedro Kourí, La Habana, Cuba.
Organización Panamericana de la Salud, Representación en La Habana, Cuba.
BioCubaFarma, Dirección de Política Científica, La Habana, Cuba.


otorgamiento de una nueva nomenclatura para esas entidades que, según su misión, se clasifican como (2):

- centros de investigación, cuando su actividad fundamental es la investigación científica y la innovación

- centros de servicios científicos y tecnológicos, encargados de la prestación de servicios, producciones especializadas e investigaciones

- unidades de desarrollo e innovación, organizadas en forma de empresas, ${ }^{6}$ unidades presupuestadas (financiadas por el Estado) $\mathrm{u}$ otro tipo de organización; en todos los casos, su cometido es desarrollar o innovar para agregar valor a los bienes, los servicios y los procesos que ofrecen.

Las actividades de investigacióndesarrollo están organizadas sobre la base de programas y proyectos (célula básica de la investigación) y se clasifican como (1):

- proyectos asociados a programas de ciencia, tecnología e innovación dirigidos a estudiar problemas de interés nacional — controlados por el CITMA, independientemente del sector de que se trate-; los programas agrupan proyectos que se interrelacionan para satisfacer las prioridades establecidas por el Estado; el sector de la salud responde por los programas de su ámbito a través de las unidades gestoras

- proyectos no asociados a programas, dirigidos a solventar las prioridades nacionales que no requieren de la implementación de un programa

- proyectos institucionales, orientados a investigar las prioridades identificadas por entidades no empresariales para la solución de problemas concretos

- proyectos empresariales, dirigidos a solucionar problemas prioritarios específicos de las empresas o los grupos empresariales.

El 26\% del total de las instituciones cubanas dedicadas a la ciencia se ocupa de la investigación, el desarrollo y la innovación para la salud y pertenece al Ministerio de Salud Pública (MINSAP),

\footnotetext{
La empresa estatal es una entidad con personalidad jurídica propia, creada para la producción de bienes y servicios mediante la administración eficiente de los recursos financieros, materiales y humanos. Se considera el eslabón fundamental de la economía (3).
}

el Grupo de las Industrias Biotecnológica y Farmacéutica (BioCubaFarma), las universidades y otros sectores (4). El MINSAP decide las prioridades del sector en armonía con los lineamientos de la política económica y social del país (5).

El Estado provee financiamiento para ejecutar los programas y los proyectos de investigación, innovación y desarro1lo, a todos los niveles. En el 2016, el presupuesto destinado por el Estado a la ciencia y la tecnología en el sector de la salud ocupó el 35,4\% del total asignado a la ciencia y la tecnología en el país (6). Otras fuentes de financiamiento son las empresas, los préstamos bancarios, las agencias internacionales, las organizaciones no gubernamentales, las donaciones y los convenios de colaboración con otros países (1).

En el informe del 2013, la Directora de la Organización Mundial de la Salud (OMS) expresó: "Todas las naciones deberían ser productoras de investigaciones tanto como consumidoras [...]. Sin embargo, [...] es necesario sobre todo colmar la brecha existente entre los conocimientos y la acción" (7).

En Cuba se ha trabajado sistemáticamente en ese sentido. El Sistema de Ciencia e Innovación Tecnológica para la Salud (SCITS) es único e integral; como principios, concibe la interdependencia entre la docencia, los servicios y la investigación, y la incorporación de los avances científicos a la práctica social (8). Este enfoque permite estudiar los problemas y las necesidades de salud en los tres niveles de atención (primario, secundario y terciario), con alternativas de solución de acuerdo con una perspectiva sectorial, intersectorial, multidisciplinaria y con apego a la cultura local (9).

El desarrollo actual del SCITS se debe a la voluntad y la atención prioritaria que el Estado le otorga a la investigación, la innovación y el desarrollo de productos y servicios para satisfacer las necesidades de salud de la población. Por ello, se garantizan las condiciones, los recursos y la formación del capital humano, de manera que en sus programas y proyectos se desarrollen acciones que contribuyan al bienestar de la población, la equidad y la eficiencia (10).

El objetivo de este artículo es describir las características del SCITS cubano e ilustrar mediante los resultados de investigaciones, su efecto positivo en la salud de la población.

\section{SISTEMA DE CIENCIA E INNOVACIÓN TECNOLÓGICA PARA LA SALUD EN CUBA}

El SCITS está organizado en 37 entidades de ciencia e innovación tecnológica: 16 centros de investigación, 3 de servicios científico-tecnológicos y 18 unidades de desarrollo e innovación que se subordinan en lo administrativo al MINSAP y metodológicamente al CITMA. Coadyuvan a su funcionamiento las sociedades científicas de la salud y otras organizaciones enfocadas en la innovación tecnológica para sustituir importaciones (e.g., la Asociación Nacional de Innovadores y Racionalizadores, las Brigadas Técnicas Juveniles y el Fórum de Ciencia y Técnica). Este sistema cuenta con más de 20000 trabajadores de diferentes categorías ocupacionales (cuadro 1). Además de la investigación, estos centros imparten docencia de pre- y posgrado y brindan servicios especializados a la población. Acorde con la política del CITMA, el MINSAP llevó a cabo un análisis de la situación de la ciencia y -en función de los cambios en el estado de salud de la población, la disponibilidad de recursos y su necesaria optimización- concentró las prioridades de investigación del período 2012-2016 en cinco programas nacionales (cuadro 2), cuya justificación es la siguiente (11):

Programa 1: Determinantes de salud, riesgos y prevención de enfermedades en los grupos vulnerables. La irrupción de nuevos actores económicos y las diferencias en los ingresos entre los diferentes grupos sociales, unidas a la situación demográfica de baja fecundidad y natalidad, el envejecimiento poblacional y la migración, imponen al Sistema Nacional de Salud (SNS) un nuevo contexto sociosanitario, donde pueden verse afectados determinados grupos sociales.

Programa 2: Organización, eficiencia y calidad en los sistemas y servicios de salud. Las limitaciones impuestas a Cuba para acceder a mercados con condiciones favorables (12) y la crisis económica mundial han precisado al SNS a priorizar y optimizar los procesos de organización, calidad y eficiencia para la sostenibilidad de las prestaciones actuales, a partir de sus concepciones y realidades.

Programa 3: Medicina natural y tradicional. Trata de preservar la cultura para la atención a la población mediante la integración de técnicas tradicionales y modernas de esta disciplina. 
CUADRO 1. Entidades de ciencia e innovación tecnológica en el sector de la salud, según su tipo y misión. Cuba, 2016a

\begin{tabular}{|c|c|c|c|}
\hline Tipo & Total & Institución ${ }^{b}$ & Misión \\
\hline Centros de investigación & 16 & $\begin{array}{l}\text { Instituto Nacional de Angiología y Cirugía Vascular } \\
\text { Instituto Nacional de Endocrinología y Enfermedades Metabólicas } \\
\text { Instituto Nacional de Higiene, Epidemiología y Microbiología } \\
\text { Instituto Nacional de Salud de Ios Trabajadores } \\
\text { Instituto de Cardiología y Cirugía Cardiovascular } \\
\text { Instituto de Gastroenterología } \\
\text { Instituto de Hematología e Inmunología } \\
\text { Instituto de Medicina Tropical Pedro Kourí } \\
\text { Instituto de Nefrología Dr. Abelardo Buch López } \\
\text { Instituto de Neurología y Neurocirugía Profesor Dr. José Rafael Estrada González } \\
\text { Instituto de Oncología y Radiobiología } \\
\text { Centro Nacional de Cirugía de Mínimo Acceso } \\
\text { Centro Nacional de Genética Médica } \\
\text { Centro Internacional de Restauración Neurológica } \\
\text { Centro de Investigaciones sobre Longevidad, Envejecimiento y Salud } \\
\text { Centro para la Investigación y Rehabilitación de las Ataxias Hereditarias } \\
\text { Carlos J. Finlay }\end{array}$ & $\begin{array}{l}\text { Investigaciones básicas y aplicadas relacionadas } \\
\text { con la carga de mortalidad, morbilidad, riesgos y } \\
\text { daños a la salud, según las especialidades }\end{array}$ \\
\hline \multirow{3}{*}{$\begin{array}{l}\text { Centros de servicios } \\
\text { científicos y tecnológicos }\end{array}$} & 3 & Centro Nacional Coordinador de Ensayos Clínicos & Vigilancia y control de los ensayos clínicos \\
\hline & & Centro Nacional de Información de Ciencias Médicas & $\begin{array}{l}\text { Investigaciones dirigidas al fortalecimiento del } \\
\text { Sistema de Información Científico-Técnica y en el } \\
\text { campo de las ciencias de la información en la salud }\end{array}$ \\
\hline & & Centro para el Control Estatal de Medicamentos, Equipos y Dispositivos Médicos & Entidad reguladora nacional \\
\hline $\begin{array}{l}\text { Unidades de desarrollo } \\
\text { e innovación }\end{array}$ & 18 & $\begin{array}{l}\text { Cardiocentro Ernesto Che Guevara (Villa Clara) } \\
\text { Cardiocentro Pediátrico William Soler } \\
\text { Centro de Desarrollo de las Ciencias Sociales y Humanísticas } \\
\text { Centro de Inmunología y Productos Biotecnológicos } \\
\text { Centro de Investigación y Referencia de la Ateroesclerosis } \\
\text { Centro de Toxicología y Biomedicina } \\
\text { Escuela Nacional de Salud Pública } \\
\text { Hospital Clínico Quirúrgico Hermanos Ameijeiras } \\
\text { Hospital Clínico Quirúrgico Juan Bruno Zayas (Santiago de Cuba) } \\
\text { Hospital General Universitario Dr. Gustavo Aldereguía Lima (Cienfuegos) } \\
\text { Hospital Pediátrico Universitario Juan Manuel Márquez } \\
\text { Hospital Pediátrico Universitario William Soler } \\
\text { Hospital Universitario Dr. Antonio Luaces Iraola (Ciego de Ávila) } \\
\text { Instituto Cubano de Oftalmología Ramón Pando Ferrer } \\
\text { Laboratorio Central de Farmacología } \\
\text { Laboratorio de Líquido Cefalorraquídeo } \\
\text { Unidad de Investigaciones Biomédicas de Villa Clara } \\
\text { Unidad de Toxicología Experimental (Villa Clara) }\end{array}$ & $\begin{array}{l}\text { Investigaciones en sistemas y servicios de salud } \\
\text { y tecnologías }\end{array}$ \\
\hline
\end{tabular}

a Recursos humanos, según la categoría ocupacional: 20171 profesionales de la salud.

' Ubicación: La Habana (entre paréntesis otra ubicación).

Fuente: Elaboración de los autores a partir de los registros de la Dirección Nacional de Ciencia y Técnica del Ministerio de Salud Pública.

Programa 4: Cáncer. Esta es la segunda causa de mortalidad en la población; este grupo de enfermedades ocasiona alrededor de 18 y 19 años de vida potencialmente perdidos en las mujeres y los hombres, respectivamente (13). Las investigaciones están dirigidas a mejorar la prevención, el diagnóstico, la atención integral y la rehabilitación.

Programa 5: Envejecimiento, longevidad y salud. Las personas con 60 años o más representan el 19\% de la población y la tendencia de este indicador es al incremento (13), lo que significa una mayor carga sobre los servicios sociales y de salud; las investigaciones deben contribuir a lograr un envejecimiento saludable.

Al cierre del 2016, se estaban ejecutando más de 2000 investigaciones, la mayoría de las cuales eran proyectos institucionales de los servicios primarios y secundarios de atención; 401 estudios de los cinco programas nacionales estaban más vinculados al nivel terciario y a instituciones de La Habana, capital del país. Los resultados alcanzados permitieron identificar nuevas prioridades de estudio para el año 2017, como la mortalidad prematura y los accidentes; la necesidad de profundizar en la investigación en ciencias básicas y medicina de avanzada (robótica, informática, nanotecnología); la evaluación de tecnologías sanitarias; y la acreditación de la calidad de las instituciones de salud.?

\footnotetext{
Morales I. Discurso inaugural por el Día de la Ciencia, La Habana, 15 de enero del 2017.
}

La concepción del proceso de investigación para la salud en Cuba sigue los cánones universales: a partir de un problema -y en correspondencia con el ambiente socioeconómico, las condiciones sanitarias, los principios y los valores de la sociedad - se identifica la idea científica, se define el problema y el método, se elabora el proyecto, se ejecuta, y se evalúan los resultados para su posterior introducción y generalización en la práctica social, hasta que concluye la intervención con nuevas interrogantes (14) (figura 1).

La conducción ética de la investigación y su rigor metodológico es responsabilidad de los investigadores, los jefes de proyecto y los directores de las instituciones, asesorados por los consejos científicos y los comités de ética de la 
CUADRO 2. Programas nacionales de ciencia, tecnología e innovación en la salud. Cuba, 2012-2016

\begin{tabular}{|c|c|c|}
\hline Programa (año de creación) & Unidad gestora & Líneas principales de investigación \\
\hline $\begin{array}{l}\text { 1. Determinantes de salud, riesgos } \\
\text { y prevención de enfermedades } \\
\text { en los grupos vulnerables (2012) }\end{array}$ & $\begin{array}{l}\text { Instituto Nacional de Higiene, } \\
\text { Epidemiología y Microbiología }\end{array}$ & $\begin{array}{l}\text { - Salud maternoinfantil } \\
\text { - Demografía (natalidad y envejecimiento) } \\
\text { - Enfermedades no transmisibles } \\
\text { - Enfermedades transmisibles } \\
\text { - Ambiente, familia y comunidad } \\
\text { - Seguridad alimentaria y nutricional } \\
\text { - Lesiones autoinfligidas } \\
\text { - Salud mental }\end{array}$ \\
\hline $\begin{array}{l}\text { 2. Organización, eficiencia y calidad } \\
\text { en los sistemas y servicios (2012) }\end{array}$ & $\begin{array}{l}\text { Escuela Nacional de Salud } \\
\text { Pública }\end{array}$ & $\begin{array}{l}\text { - Gestión en la salud y sus componentes: equidad, accesibilidad, eficiencia, eficacia, participación } \\
\text { - Pocial, comunitaria e intersectorial } \\
\text { - Cerfeccionamiento de la gestión de riesgo en las funciones reguladoras } \\
\text { - Aplicaciones de las tecnologías de la información y las comunicaciones } \\
\text { - Satisfacción del personal de salud }\end{array}$ \\
\hline $\begin{array}{l}\text { 3. Medicina natural y tradicional } \\
\text { (2015) }\end{array}$ & $\begin{array}{l}\text { Universidad de Ciencias } \\
\text { Médicas de La Habana }\end{array}$ & $\begin{array}{l}\text { - Seguridad, efecto y calidad de la medicina natural y tradicional en las enfermedades crónicas } \\
\text { no transmisibles y la atención primaria de salud } \\
\text { - Ensayos clínicos de estas modalidades aprobadas por el Sistema Nacional de Salud } \\
\text { - Utilización de medicamentos herbarios y otras formas de medicina natural y tradicional aprobadas } \\
\text { - Estudios de sostenibilidad y factibilidad de tecnologías } \\
\text { - Evaluación, registro y control de productos naturales, equipos y procedimientos de la medicina } \\
\text { natural y tradicional }\end{array}$ \\
\hline 4. Cáncer (2016) & $\begin{array}{l}\text { Instituto Nacional de } \\
\text { Oncología y Radiobiología }\end{array}$ & $\begin{array}{l}\text { - Nuevos marcadores tumorales y productos para el diagnóstico y el tratamiento } \\
\text { - Inmunología e inmunoterapia } \\
\text { - Ensayos clínicos con productos nuevos o en nuevas combinaciones para el tratamiento } \\
\text { - Incidencia y mortalidad por cáncer cervicouterino en las mujeres en edad fértil } \\
\text { - Prevención y educación para la salud, el autocuidado y los signos de alarma del cáncer con } \\
\text { intervenciones, desde edades tempranas } \\
\text { - Evaluación de tecnologías sanitarias en las áreas de cirugía, radioterapia, medicina nuclear e } \\
\text { imagenología }\end{array}$ \\
\hline $\begin{array}{l}\text { 5. Envejecimiento, longevidad } \\
\text { y salud (2016) }\end{array}$ & $\begin{array}{l}\text { Centro de Investigaciones } \\
\text { de la Tercera Edad }\end{array}$ & $\begin{array}{l}\text { - Biomarcadores del envejecimiento normal y patológico } \\
\text { - Evaluación del desempeño físico, del manejo de la fragilidad y de los síndromes geriátricos } \\
\text { - Enfermedades asociadas al envejecimiento: factores de riesgo, causas, carga de enfermedad, } \\
\text { efecto, costos, detección oportuna, diagnóstico y tratamiento } \\
\text { - Discapacidad, dependencia y entornos amigables } \\
\text { - Atención y cuidado para adultos mayores, familiares y cuidadores }\end{array}$ \\
\hline
\end{tabular}

Fuente: Elaboración de los autores a partir de la referencia 11.

FIGURA 1. Proceso de investigación en la salud. Cuba, 2016

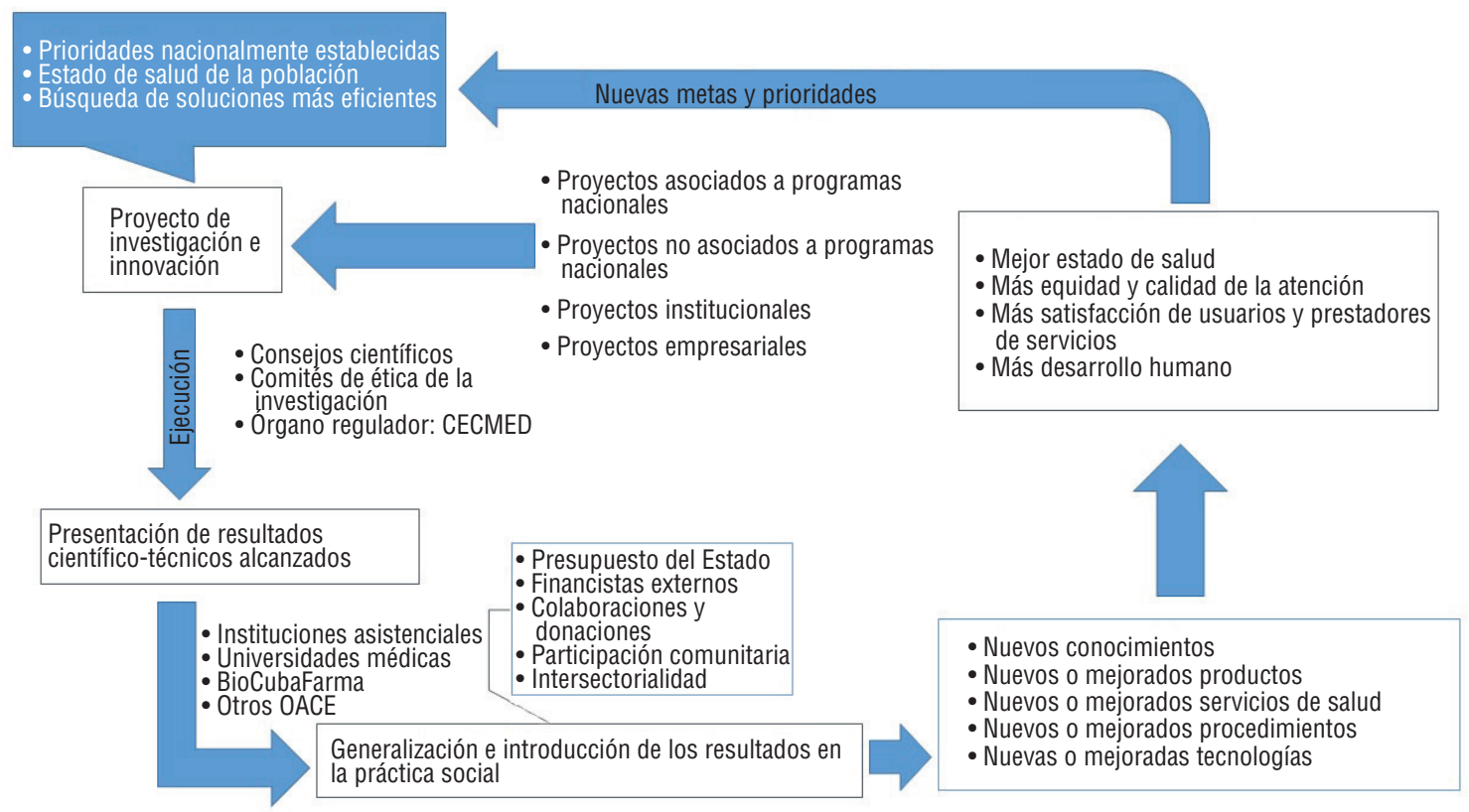

Nota: CECMED: Centro para el Control Estatal de Medicamentos, Equipos y Dispositivos Médicos; BioCubaFarma: Grupo de las Industrias Biotecnológica y Farmacéutica; OACE: Organismo de la Administración Central del Estado.

Fuente: Elaboración de los autores. 
investigación (1, 2). A nivel nacional le corresponde al Centro para el Control Estatal de Medicamentos, Equipos y Dispositivos Médicos (CECMED) garantizar la seguridad y el cumplimiento de las regulaciones deontológicas en las personas y las comunidades.

En los proyectos relacionados con productos y servicios (medicamentos, tecnologías y programas), la concepción es de ciclo cerrado.

En el 2012 se creó BioCubaFarma (15, 16), organización superior de dirección, subordinada al Consejo de Ministros, que integra los centros de investigacióndesarrollo-producción de la biotecnología, la industria farmacéutica y los equipos médicos de alta tecnología. BioCubaFarma —con sus 32 empresas, 70 instalaciones productivas, 10 centros de investigación, 11 unidades de investigación y desarrollo y un centro de servicios científicotecnológicos, en los que laboran 21000 trabajadores - también forma parte del SCITS. Su objetivo es garantizar al SNS los medicamentos esenciales (biotecnológicos y genéricos), los productos, las materias primas, las vacunas, los reactivos, los medios diagnósticos y los equipos requeridos, además de tener sus propias líneas de investigación $(17,18)$ (cuadro 3).

BioCubaFarma se autofinancia y concibe la investigación científica no solo como productora de conocimientos, sino como fuente de recursos materiales y financieros, a fin de recuperar la inversión sin eludir su compromiso social con la salud pública.

La integración entre los centros de investigación y de prestación de servicios científico-tecnológicos, las unidades de desarrollo e innovación, las universidades y las instituciones asistenciales permite a la población cubana el acceso a una medicina altamente calificada. También facilita que los medicamentos, los servicios y los productos de alta tecnología sean accesibles a los grupos poblacionales que los requieran, sin costos tangibles y con un efecto positivo para su salud.

\section{Desarrollo del capital humano}

Para el desarrollo de la actividad científica es imprescindible contar con un personal altamente calificado y comprometido con los problemas de salud y su solución (10). La formación para la investigación se inicia en los estudios de pregrado, se fortalece en el desempeño de la actividad laboral y alcanza su mayor grado de actualización y especialización en la educación posgraduada (en Cuba o en el extranjero), que llega hasta el nivel de doctorado $(10,19)$.

Los profesionales dedicados a la investigación se categorizan mediante un sistema que integra los años de experiencia, el nivel profesional y los resultados, con evaluaciones anuales que muestren los avances o los retrocesos en los indicadores que se van a medir a nivel individual e institucional, como la participación en proyectos y eventos científicos, las publicaciones, la conducción de tesis, la obtención de premios y grados científicos, los registros de patentes, el derechos de autor y la introducción de resultados (19).

Al cierre del 2016, el MINSAP contaba con 3214 investigadores categorizados como titulares $(8,5 \%)$, auxiliares $(21,7 \%)$, agregados $(57,6 \%)$ y aspirantes $(12,2 \%)$. En el año se produjeron 224 bajas (por migración, fallecimiento o jubilación); el análisis muestra los porcentajes más bajos de investigadores en las categorías de titulares y aspirantes (20).

La extensión de la investigación a todas las unidades de salud ha traído consigo el desafío de un nuevo proceso de "alfabetización" (21) para el uso adecuado de los mecanismos y los instrumentos de la ciencia, dada la necesidad de enfrentar las dificultades que son comunes para los SCITS

CUADRO 3. Productos suministrados por el Grupo de las Industrias Biotecnológica y Farmacéutica BioCubaFarma al Sistema Nacional de Salud. Cuba, 2016

\begin{tabular}{lc}
\hline \multicolumn{1}{c}{ Producto } & Total \\
\hline Medicamentos (biotecnológicos y genéricos) & 499 \\
Reactivos & 64 \\
Medios de diagnóstico & 135 \\
Equipos & 76 \\
Productos para uso estomatológico & 24 \\
Materias primas para la asistencia médica & 12 \\
Materias primas para la medicina natural tradicional y químico-dispensarial & 53 \\
Total & 863 \\
\hline
\end{tabular}

Fuente: Elaboración de los autores a partir de las referencias 17 y 18. de la Región de las Américas, como el desarrollo de competencias de investigación, la participación consciente y responsable de las comunidades afectadas y de otros sectores, y el incremento de las publicaciones y las patentes como forma de validar los resultados (22).

Otros retos son el acceso al financiamiento y su utilización correcta, la obsolescencia tecnológica, el mantenimiento de las instalaciones, la inestabilidad y el envejecimiento de los investigadores, así como la insuficiente importancia que algunos decisores confieren a la investigación y a la innovación $(7,22)$.

\section{INVESTIGACIONES Y SU EFECTO EN LA SALUD DE LA POBLACIÓN}

Para ilustrar cómo han influido en la salud las investigaciones del SCITS cubano, se han seleccionado algunos ejemplos de investigación-desarrollo por sus aportes demostrados al estudio y el control de los problemas de salud priorizados, y que combinan investigaciones básicas y aplicadas, desde el enfoque de las ciencias biomédicas, sociales y otras (cuadro 4).

Uno de esos ejemplos es la generación de productos - medicamentos, reactivos, etc.- y tecnologías -equipos y sistemas- para la salud, algo que en Cuba resulta vital para disminuir la mortalidad por enfermedades, principalmente las que ocupan los tres primeros lugares como causa de muerte: enfermedades del corazón (217,7 fallecidos por 100000 habitantes), tumores malignos (216,3 por 100000 habitantes), enfermedades cerebrovasculares $(84,2$ por 100000 habitantes) y diabetes mellitus (20,0 por 100000 habitantes) (13).

También las investigaciones sobre el dengue y la diabetes, y el estudio biopsicosocial de la discapacidad abarcan los sistemas y los servicios de la salud, y han generado cambios en la práctica médica, las políticas sociales y los programas específicos de educación y atención a los grupos vulnerables.

\section{Generación de productos y tecnologías para la salud}

El primer resultado de este tipo de investigaciones fue el Sistema Ultramicroanalítico (SUMA), que ha permitido el tamizaje masivo de varias enfermedades infecciosas en la población general y 


\section{CUADRO 4. Algunas investigaciones y su efecto en la salud de grupos poblacionales. Cuba, 2016}

\begin{tabular}{|c|c|c|}
\hline Problema de salud & Resultados de la investigación & Efecto en la salud de grupos poblacional \\
\hline $\begin{array}{l}\text { Defectos congénitos, infección por } \\
\text { el VIH y hepatitis B en las } \\
\text { embarazadas }\end{array}$ & $\begin{array}{l}\text { Desarrollo del Sistema Ultramicroanalítico } \\
\text { (SUMA) para el tamizaje prenatal, } \\
\text { disponible en hospitales, bancos de sangre } \\
\text { e institutos de investigación }\end{array}$ & $\begin{array}{l}\text { - Se realizaron } 4098860 \text { pruebas de alfafetoproteína; se de } \\
\text { 6,4\%; hubo reducción de la mortalidad infantil en 2,1 por } \\
\text { cobertura: más del } 98 \% \text { de las gestantes del país } \\
\text { - Hepatitis B: } 1824520 \text { pruebas; } 1,4 \% \text { positivas } \\
\text { - VIH 1+2: } 5818895 \text { pruebas; 0,2\% positivas; la OMS valid } \\
\text { eliminación de la transmisión vertical del VIH y la sífilis en }\end{array}$ \\
\hline Enfermedades congénitas & $\begin{array}{l}\text { Desarrollo de la tecnología SUMA, para el } \\
\text { tamizaje neonatal, disponible en hospitales, } \\
\text { bancos de sangre e institutos de } \\
\text { investigación }\end{array}$ & $\begin{array}{l}\text { Hipotiroidismo congénito (801 casos detectados en } 3350373 \\
\text { fenilcetonuria ( } 20 \text { casos en } 1055575 \text { muestras), hiperplasia sL } \\
780771 \text { ), déficit de biotinidasa ( } 5 \text { en } 759 \text { 935) y galactosemia }\end{array}$ \\
\hline $\begin{array}{l}\text { Transmisión transfusional de la } \\
\text { infección por el VIH y las hepatitis } \\
\text { B y C; tamizaje masivo y control } \\
\text { epidemiológico }\end{array}$ & $\begin{array}{l}\text { Desarrollo de la tecnología SUMA para la } \\
\text { certificación de las donaciones de sangre y el } \\
\text { control epidemiológico, disponible en } \\
\text { hospitales, bancos de sangre e institutos de } \\
\text { investigación }\end{array}$ & $\begin{array}{l}\text { Donantes de sangre: } \\
\text { - VIH 1+2: } 12798394 \text { pruebas; 0,7\% positivas } \\
\text { - Hepatitis B: } 12074614 \text { pruebas; 0,9\% positivas } \\
\text { - Hepatitis C: } 10017461 \text { pruebas; 0,9\% positivas } \\
\text { Control epidemiológico: } \\
\text { - VIH 1+2: } 35514618 \text { pruebas; } 0,6 \% \text { positivas } \\
\text { - Hepatitis B: } 9365416 ; 1,2 \% \text { positivas } \\
\text { - Hepatitis C: } 2226778 \text { pruebas; } 5,3 \% \text { positivas } \\
\text { - Dengue (IgM): } 980104 \text { pruebas; } 32,7 \% \text { positivas } \\
\text { - Enfermedad de Chagas: } 14213 \text { pruebas; } 4,8 \% \text { positivas } \\
\text { - Lepra: } 31725 \text { pruebas; } 10,6 \% \text { positivas }\end{array}$ \\
\hline
\end{tabular}

Enfermedades transmisibles prevenibles por vacunación

Alta mortalidad por infarto agudo de miocardio

Alta incidencia de cardiopatía isquémica

Neutropenia en pacientes oncohematológicos, con VIH/sida, o secundaria al tratamiento con quimio- 0 radioterapia

Mortalidad por cáncer de pulmón de células no pequeñas

Úlcera del pie diabético y amputaciones

Diabetes y autocuidado

Programa Nacional de Educación al Paciente Diabético y su implementación

Identificación de los signos de alarma tempranos de la enfermedad

Caracterización de la magnitud y el alcance del problema

combinada contra la difteria, el tétanos, la epidérmico recombinante aplicado mediante inyección intralesional socioeconómica de las personas

con discapacidad
Registrada en el 2010; simplificó el Programa Nacional de Inmunización al reducir de once a seis el número de vacunaciones. La cobertura de vacunación es superior al $98,7 \%$

Campaña de vacunación masiva en 1989. Se incluyó en el Programa Nacional de Inmunización desde 1991. La incidencia se redujo de 14 por 100000 habitantes en la década de 1980 a menos de 0,2 por 100000 habitantes a partir del 2006

Desde su introducción en 1991, disminuyó la mortalidad hospitalaria por infarto agudo de miocardio entre $50 \%$ y $70 \%$; el medicamento está disponible en centros de atención primaria de salud con unidades de cuidados progresivos

Tratamiento de la hipercolesterolemia (factor de riesgo coronario); incorporado al CBM desde 1991, está disponible en las farmacias

Incorporado al CBM y disponible en las instituciones hospitalarias desde el 2004; eficaz en la recuperación del conteo absoluto de neutrófilos en pacientes oncohematológicos (adultos y pediátricos) y con VIH/sida; disminución de la estadía hospitalaria; disminución del riesgo de infecciones asociadas; mejoría de la calidad de vida

Inmunoterapia activa; incorporada al CBM; aumento de la supervivencia y la calidad de vida

Más de 20000 pacientes diabéticos tratados en más de 85 instituciones de salud (hasta el 2014); establecimiento del Programa de Atención Integral al Paciente con Úlcera del Pie Diabético (incluye uso del producto) que coordina acciones de los niveles primario y secundario de salud; incorporado al CBM desde el 2007; se logró $81,2 \%$ de reducción del índice de amputaciones (12 000 amputaciones evitadas)

Disminución de la dosis de medicamentos en pacientes no dependientes de insulina; reducción de los niveles de $\mathrm{HbA}$ de $12 \%$ a 7,6\%, de la frecuencia de sobrepeso corporal de $77 \%$ a $20 \%$ de los casos y de las hospitalizaciones promedio anuales por descompensación metabólica en diabéticos tipo 1, de 2 a 3 por persona a menos de 1

Incorporados a las normas para el manejo terapéutico en las guías de Cuba, la OMS y otros países de la Región; clasificación de casos: $96 \%$ de sensibilidad y 100\% de especificidad

Se estudiaron 366864 personas con discapacidad (prevalencia: 3,26\%); se desarrollaron programas comunitarios en genética, rehabilitación y trabajo social; se implementó la ayuda técnica, monetaria y de alimentación; se promulgó y aplicó una ley para la protección de madres con hijos afectados: desde el 2016 se han protegido 7462 madres de personas con discapacidad grave piedad Intelectual (OMPI). En la vacuna pentavalente solo se premió el componente

Haemophilus influenzae tipo b (antígeno sintético) (10).

Nota: CBM: cuadro básico de medicamentos; HbA: hemoglobina glicosilada; OMS: Organización Mundial de la Salud; VIH: infección por el virus de la inmunodeficiencia humana.

Fuente: Elaboración de los autores a partir de las referencias 10, 17, 18, 23-38. 
grupos específicos, como embarazadas, donantes de sangre, portadores de la infección por el virus de la inmunodeficiencia humana (VIH) (10). Hasta septiembre del 2014, se habían realizado más de 48 millones de pruebas mediante el SUMA para el control de la trasmisión del VIH, las hepatitis B y C, el dengue y las enfermedades de Chagas y de Hansen (lepra). En los donantes de sangre, se hicieron más de 34 millones de pruebas para el control de la transmisión del VIH y las hepatitis B y C (23).

En el marco del Programa Nacional de Atención Materno Infantil se estudiaron más de 4 millones de gestantes hasta el 2014. La detección prenatal de defectos congénitos mediante la cuantificación de alfafetoproteína en el suero materno ha permitido ofrecer a las gestantes y sus parejas la realización de estudios adicionales, el asesoramiento genético o la terminación selectiva e informada de la gestación, pues mediante estas técnicas se pueden confirmar defectos congénitos graves en el feto (24). Se han realizado, además, más de 7 millones de pruebas para el control de la trasmisión vertical del VIH, la sífilis y la hepatitis B a las gestantes y sus parejas, lo que contribuyó al logro de la designación de Cuba como el primer país del mundo en eliminar la transmisión vertical del VIH y la sífilis congénita (25). En la actualidad, este programa cubre la atención de más del 95\% de las gestantes del país (24).

Las investigaciones también han facilitado la producción nacional de 8 de las 11 vacunas que integran el Programa Nacional de Inmunización, cuya cobertura es superior al 98\%. Gracias a ello, enfermedades como el tétanos, la meningitis meningocócica, la infección por Haemophilus influenzae tipo b y la fiebre tifoidea, han dejado de ser un problema de salud en Cuba, mientras la morbilidad y la mortalidad por hepatitis B se han reducido en más del 98\% (26).

Además de las vacunas preventivas, la biotecnología entrega al SNS otros productos, como biofármacos para el tratamiento de enfermedades no transmisibles (cardiovasculares, oncológicas y diabetes, entre otras). Otro ejemplo es el grupo de tecnologías desarrolladas para el tamizaje, la detección y el tratamiento temprano del cáncer cervicouterino, suministrado al SNS desde el 2010; entre los años 2012 y 2014 se analizó el $71,8 \%$ de las mujeres entre 25 y 64 años, lo que significó un avance respecto al 2009. Sin embargo, ese porcentaje quedó por debajo del $80 \%$ de cobertura recomendado por la OMS para evaluar si estas intervenciones han incidido en la disminución de la mortalidad por esta neoplasia (39).

Los productos y las tecnologías de BioCubaFarma forman parte del Cuadro Básico de Medicamentos (17) y otras aplicaciones del MINSAP, por lo que reducen significativamente las importaciones.

\section{Educación de las personas con diabetes}

Las investigaciones e intervenciones acerca de los conocimientos, las actitudes y las prácticas de las personas con diabetes, iniciadas en el Instituto Nacional de Endocrinología, han sido un pilar fundamental del Programa Nacional de Atención y Educación al Paciente Diabético, implementado en 1981 (31). Sus resultados contribuyen a evitar las complicaciones y elevar la calidad de vida de los enfermos, que han podido prescindir del uso de nuevos tipos de insulina $\mathrm{u}$ otras tecnologías. Aunque se ha introducido el Heberprot- $\mathrm{P}^{\circledR}$ como tratamiento eficaz de las úlceras del pie diabético (30), se demostró que solo con el cumplimiento estricto de las normas terapéuticas e higiénicas contenidas en el programa, el paciente puede obtener el máximo beneficio del producto.

Las investigaciones mostraron un aumento de los conocimientos y las destrezas de los pacientes para el cuidado diario (independientemente de la edad, el grado de escolaridad, el tipo y la duración de la diabetes), asociado a una notable disminución de las cifras promedio de hemoglobina glicosilada $\left(\mathrm{HbA}_{1}\right)$, y la reducción de las dosis de medicamentos, la frecuencia de sobrepeso corporal y el número promedio de hospitalizaciones por descompensación $(31,32)$.

En los pacientes con diabetes tipo 1 de inicio reciente, se estudió la aparición y el desarrollo de las complicaciones a largo plazo. Se encontró que la frecuencia de retinopatía diabética había sido significativamente menor en el grupo seguido con una metodología interactiva — basada en el diálogo constructivo entre quien emite la información y quien la recibe; de esta forma, se escuchan criterios, se desarrollan habilidades para afrontar las diferentes exigencias terapéuticas y se trabaja para desarrollar motivaciones y autoconfianza para afrontar los problemas-, con respecto a los pacientes que solo recibían educación individual mediante la consulta médica tradicional. Un resultado similar se obtuvo cuando se evaluó la evolución de la nefropatía y la neuropatía periférica (31).
Lo expuesto no minimiza la magnitud de la diabetes como problema de salud, por lo que se debe continuar trabajando en la comunidad con los pacientes y la población general para su prevención, mediante el cumplimiento correcto del tratamiento médico e higiénico-sanitario (32). Sigue siendo fundamental la divulgación de la importancia de la autorresponsabilidad en la salud (40).

\section{Investigaciones acerca del dengue}

Estos estudios alcanzaron su máxima expresión en 1981, con la epidemia letal de dengue hemorrágico que afectó al país (41). Los primeros esfuerzos estuvieron dirigidos a la epidemiología de la enfermedad, y a la caracterización de la evolución clínica de los niños fallecidos por dengue grave y sus signos de alarma, definidos como "un grupo de signos y síntomas que anuncian la inminencia del choque por dengue" (36). Estos trabajos recibieron el reconocimiento de la Organización Panamericana de la Salud (OPS) y la OMS como la pauta para identificar tempranamente a los pacientes que van a evolucionar hacia el dengue grave, quienes requieren de una atención terapéutica diferenciada (33-35). El estudio prospectivo DENCO (36), que abarcó a más de 1700 casos confirmados, mostró que la clasificación basada en los signos de alarma inicialmente empleada en Cuba tenía $96 \%$ de sensibilidad y 100\% de especificidad, por lo que se incorporó a los documentos normativos de la OMS y a las guías cubanas y de varios países de la Región para el manejo integral de los pacientes con dengue.

Entre estas investigaciones, lideradas por el Instituto de Medicina Tropical Pedro Kourí, se destacan el establecimiento de la hipótesis integral sobre el dengue hemorrágico (41) y los estudios virológicos para la caracterización de los virus causantes de las epidemias y los brotes de esta enfermedad en Cuba, y los marcadores de virulencia y su asociación con el dengue grave. Estas investigaciones también han beneficiado a otros países de la Región.

Los estudios entomológicos y las investigaciones operacionales para el control del mosquito Aedes aegypti han permitido caracterizar e identificar los principales sitios de cría y los mecanismos de resistencia del vector. Asimismo, BioCubaFarma y otros centros trabajan para la obtención de un candidato vacunal contra esta enfermedad.

También son relevantes los estudios sobre los determinantes socioeconómicos y 
la participación social e intersectorial, así como los relacionados con los conocimientos, las actitudes y las prácticas de la población. Pese a las medidas tomadas por el MINSAP y el apoyo estatal, al cierre del 2016 existían municipios con índices de infestación ${ }^{8}$ por encima del nivel permisible para el control de epidemias establecido en el Programa Nacional de Erradicación del Aedes aegypti $(0,05)$, lo que confirma la importancia de los determinantes socioeconómicos para el control de la enfermedad. Actualmente estas investigaciones abarcan el estudio de otras arbovirosis causadas por el mismo vector (42).

\section{Estudio biopsicosocial de las personas con discapacidad}

Las personas con discapacidad constituyen uno de los sectores más vulnerables en todas las sociedades; en la actualidad, su atención y estudio son un desafío en cualquier país. En Cuba se diseñó y realizó el primer estudio integral de personas con discapacidad entre los años 2001 y 2003 (37).

Se estudiaron 366864 personas con discapacidad (cuya prevalencia en la población examinada fue de $3,26 \%$ ). Las mayores tasas se encontraron en las provincias de Guantánamo (4,13\%) y Santiago de Cuba (3,79\%).

En la encuesta se detectaron 140489 $(38,29 \%)$ personas con discapacidad intelectual, para una prevalencia de 1,25\%. De los encuestados detectados, 92506 $(25,2 \%)$ presentaban discapacidad físicomotora, para una prevalencia de $0,82 \%$; $46455(12,7 \%)$ tenían discapacidad visual (prevalencia de 0,41\%); 36869 $(10,1 \%)$, discapacidad mental (prevalencia de $0,33 \%) ; 23620(6,4 \%)$ trastornos auditivos (prevalencia de 0,21\%); 25094 $(6,8 \%)$ presentaban discapacidades mixtas (prevalencia de 0,22\%); y 1831 (0,5\%) tenían diagnóstico de insuficiencia renal crónica (prevalencia de 0,02\%) (37).

\footnotetext{
Refleja el grado de infestación por el vector en la zona encuestada; se expresa como el porcentaje de viviendas y locales positivos con respecto al número total de viviendas inspeccionadas (42)
}

\section{CUADRO 5. Lecciones aprendidas}

Las investigaciones en la salud en Cuba y su aplicación son una realidad gracias a

- La voluntad política del Estado, que ha propiciado el financiamiento, la articulación y la generación de sinergias entre los servicios de salud, los centros de investigación, las empresas de distintos sectores y las disciplinas, lo cual permite a un país en desarrollo realizar investigaciones e introducir las tecnologías más avanzadas para elevar los estándares de salud de su población

- La existencia del Sistema de Ciencia e Innovación Tecnológica para la Salud (SCITS), ya que la definición de las prioridades de investigación-desarrollo y el aporte de los recursos mayoritarios es un compromiso del Estado

- El dinamismo del SCITS para adaptar sus prioridades, organización y funcionamiento a los cambios sociales y sanitarios

- La atención sistemática y la preparación del capital humano, el diálogo con los decisores, el rigor metodológico, el compromiso social y la ética de los investigadores, como necesidades del SCITS para cumplir sus objetivos

Entre los programas desarrollados para atender la discapacidad, de acuerdo con los resultados de la investigación, se destacan:

- el diseño de programas con base comunitaria: genética, rehabilitación y trabajo social

- el establecimiento de 23 líneas de investigación sobre los factores asociados con la discapacidad

- el reconocimiento legal de la condición de "madre cuidadora", que percibe su salario íntegro por atender en el hogar a su hijo con discapacidad

- la entrega de ayudas técnicas y económicas, y alimentación gratuita a las personas con discapacidad.

A solicitud de sus gobiernos, la investigación se replicó en Venezuela, Ecuador, Nicaragua, Bolivia, y San Vicente y las Granadinas entre el 2007 y el 2010 (43).

\section{Comentarios finales}

Las investigaciones reseñadas en cada uno de los temas seleccionados son ejemplos de que en las ciencias biomédicas, las soluciones emergen de las áreas de contacto entre las diferentes disciplinas y que "avanza no sólo el que tenga más conocimientos, sino el que mejor los combine" (21).

La consecución de los resultados alcanzados ha sido producto de un proceso largo y arduo, que abarca desde la voluntad política, el desarrollo del capital humano, el fomento de una industria nacional, y la creación de condiciones materiales y financieras para operar, hasta la necesidad de solventar contradicciones entre las posturas epistemológicas y los saberes, o entre los gestores y los investigadores (cuadro 5).

\section{CONCLUSIONES}

El SCITS cubano se sustenta en una política nacional, promueve la ciencia en todos los niveles de atención y ha solucionado los problemas de salud prioritarios. Enfrenta desafíos comunes a otros países de la Región, como el acceso al financiamiento, la obsolescencia tecnológica y la preservación del capital humano, derivados de las limitaciones económicas impuestas y de los cambios en su contexto socioeconómico.

Las investigaciones presentadas ofrecen aportes relevantes que se han introducido en la práctica sanitaria en forma de políticas, programas, productos, tecnologías, y servicios sociales y de salud de calidad. Estos avances han cubierto la población necesitada y han tenido un efecto positivo en su estado de salud. Aun así, estos y otros problemas, por su magnitud y complejidad, continuarán siendo objeto de estudio de las ciencias del campo de la salud ad infinitum.

Conflicto de intereses. Ninguno. El Dr. V. Gustavo Sierra González trabaja para BioCubaFarma.

Declaración. Las opiniones expresadas en este manuscrito son responsabilidad de los autores y no reflejan necesariamente los criterios ni la política de la Revista Panamericana de Salud Pública / Pan American Journal of Public Health o de la Organización Panamericana de la Salud.

\section{REFERENCIAS}

1. República de Cuba, Ministerio de Ciencia, Tecnología y Medio Ambiente. Resolución 44/2012. Reglamento para el proceso de elaboración, aprobación, planificación, ejecución y control de los Programas y Proyectos de Ciencia, Tecnología e Innovación. La Habana: CITMA; 2012. Disponible en: http:/ / files.sld. $\mathrm{cu}$ /sccs / files / 2012/03/ministerio-de- ciencia-tecnologia-y-medio-ambienteres-4412.pdf Acceso el 14 de abril de 2017

2. República de Cuba, Consejo de Estado. Decreto Ley No. 323. Resolución 164/2014. 
Gac Oficial Republica Cuba. 2014;29;(37): 1-14. Disponible en: http:/ /www.redciencia.cu/documentos/decretoley323.pdf Acceso el 14 de abril de 2017.

3. República de Cuba, Consejo de Estado. Decreto-Ley No. 252/07. Sobre la continuidad y el fortalecimiento del sistema de dirección y gestión empresarial cubano. Artículo 13. Gac Oficial Republica Cuba. 2007;(41 Extraordinaria del 17 de agosto):239. Disponible en: http://www.gacetaoficial. cu/ Acceso el 10 de diciembre de 2017.

4. Pérez Montoya ER. Ciencia, tecnología e innovación en Cuba. Desafíos y proyecciones. Congreso Internacional Pedagogía 2017; 2017 enero 30-febrero 3; La Habana.

5. Partido Comunista de Cuba. Actualización de los Lineamientos de la Política Económica y Social del Partido y la Revolución para el Período 2016-2021, aprobados en el 7. ${ }^{\circ}$ Congreso del Partido en abril de 2016 y por la Asamblea Nacional del Poder Popular en julio de 2016. La Habana; 2016. Disponible en: http://www.granma.cu/ file/pdf/gaceta/01Folleto.Lineamientos4.pdf Acceso el 14 de abril de 2017.

6. República de Cuba, Ministerio de Ciencia, Tecnología y Medio Ambiente. Informe de los organismos de la administración central del Estado y los órganos superiores de dirección empresarial sobre la planificación de las actividades de la Ciencia, la Tecnología y la Innovación para el año 2017. La Habana: CITMA; 2016.

7. Organización Mundial de la Salud. Investigaciones sobre una cobertura sanitaria universal. Informe sobre la Salud en el Mundo, 2013. Ginebra: OMS; 2013. Disponible en: http://apps.who.int/medicinedocs/documents/s22233es/s22233es.pdf Acceso el 21 de abril de 2017.

8. Rojas Ochoa F. Fundamentos políticoideológicos de la salud pública revolucionaria cubana. La Habana: Editorial Ciencias Médicas; 2009.

9. Rojo Pérez N, Menchaca S, Castell-Florit P, Piñero J, Barroso Z, Bayarre H. Investigaciones en sistemas y servicios de salud en Cuba y su proyección hasta el 2015. Rev Cubana Salud Publica. 2010;36(3):209-14. Disponible en: http://scielo.sld.cu/pdf/ rcsp/v36n3/spu04310.pdf Acceso el 21 de abril de 2017.

10. Organización Mundial de la Salud. Experiencia cubana en la producción local de medicamentos, transferencia de tecnología y mejoramiento en el acceso a la salud. Ginebra: OMS; 2015. Disponible en: http://www.who.int/phi/publications / Cuba_case_studySP.pdf Acceso el 21 de abril de 2017

11. República de Cuba, Ministerio de Ciencia, Tecnología y Medio Ambiente. Programas de ciencia, tecnología e innovación. La Habana: CITMA; 2015.

12. Drain PK, Barry M. Fifty years of U.S. embargo: Cuba's health outcomes and lessons. Science. 2010;30;328(5978):572-3. Disponible en: http://www.sciencemag.org/cgi/content/full/328/5978/572 Acceso el 24 de septiembre de 2017.

13. República de Cuba, Ministerio de Salud Pública, Dirección Nacional de Registros Médicos y Estadísticas de Salud. Anuario Estadístico de Salud 2016. La Habana:
MINSAP; 2017. Disponible en: http:// bvscuba.sld.cu/anuario-estadistico-decuba/ Acceso el 2 de junio de 2017.

14. Peters DH, Tran N, Adam T. Investigación sobre la implementación de políticas: guía práctica. Alianza para la investigación en política y sistemas de salud. Ginebra: Organización Mundial de la Salud; 2013.

15. República de Cuba, Consejo de Ministros. Decreto No. 307. Gac Oficial Republica Cuba. 2012;60(52):243-5. Disponible en: http://www.paho.org/cub/index.php? option=com_docman\&view=download\&category_slug $=$ cnic\&alias $=863$-biocubaf arma-gaceta-oficial\&Itemid=226 Acceso el 3 de octubre de 2017.

16. República de Cuba, Ministerio de Economía y Planificación. Resolución No. 590/12. Gac Oficial Republica Cuba. 2012;60(52):245-8. Disponible en: http:/ / www.paho.org/cub/ index.php?option=com_docman\&view=download\&category_slug=cnic\&alias=863-biocubafarma-gaceta-oficial $\&$ Itemid=226 Acceso el 3 de octubre de 2017.

17. República de Cuba, Ministerio de Salud Pública, Dirección de Medicamentos y Tecnologías. Cuadro básico de medicamentos y productos naturales 2017. La Habana: MINSAP; 2017. Disponible en: http://temas.sld.cu/medicamentosterapeutica Acceso el 11 de octubre de 2017.

18. BioCubaFarma. Catálogo comercial. La Habana: BioCubaFarma; 2017. Disponible en: http:/ / www.biocubafarma.cu/wp-content/uploads/2014/10/BCF-Catalogo.pdf Acceso el 21 de abril de 2017.

19. Navarro-Machado VR, Falcón-Hernández A, Espinosa-Brito AD, Romero-Cabrera AJ. A scientific and technological innovation system in a Cuban hospital (20002014). MEDICC Rev. 2016;18(1-2):34-40. Disponible en: http://www.medicc.org/ mediccreview $/$ index.php? issue $=38 \& i d=$ $518 \& a=v a h t m l$ Acceso el 21 de abril de 2017.

20. República de Cuba, Ministerio de Salud Pública, Dirección Nacional de Ciencia y Tecnología. Registro de cuadros. La Habana: MINSAP; 2016.

21. Lage A. Sociedad del conocimiento y soberanía nacional en el siglo XXI: el nexo necesario. La Habana: Fundación Alejo Carpentier; 2017. Disponible en: http:// www.fundacioncarpentier.cult.cu/carpentier/sociedad-del-conocimiento-y-soberania-nacional-en-el-siglo-xxi-el-nexo-necesario Acceso el 2 de octubre de 2017.

22. Castro Díaz-Balart F. Rediseñar el sistema de ciencia e innovación. Cubadebate. 2017 septiembre 6. Disponible en: http:/ / www. cubadebate.cu/opinion/2017/09/06/redisenar-el-sistema-de-ciencia-e-innovacion/\#.WeZqAXbB-M8 Acceso el 2 de octubre de 2017.

23. González Fernández RS, Arteaga Yera AL. La tecnología SUMA en el Sistema Nacional de Salud. Actas Cuba Salud 2015 Convención Internacional de Salud Pública; 2015 abril 20-24; La Habana, Cuba. La Habana: Editorial Ciencias Médicas; 2015. Disponible en: http://www.convencionsalud2015.sld.cu/index.php/convencionsalud/2015/paper/view/1089/606 Acceso el 2 de octubre de 2017.

24. Marcheco Teruel B, Lantigua Cruz A, Rojas Betancourt I, Benítez Cordero Y.
Genética médica en Cuba: sus resultados e impacto en el cuidado de la salud maternoinfantil en 35 años (1980-2014). An Acad Ciencias Cuba. 2016;6(3). Disponible en: http://www.revistaccuba.cu/index. $\mathrm{php} / \mathrm{acc} /$ article / view File / 585/508 Acceso el 2 de octubre de 2017.

25. Organización Mundial de la Salud. La OMS valida la eliminación en Cuba de la transmisión de madre a hijo del VIH y de la sífilis (comunicado de prensa, $2015 \mathrm{ju}$ nio 30). Ginebra: OMS; 2015. Disponible en: http://www.who.int/mediacentre/ news/releases/2015/mtct-hiv-cuba/es / Acceso el 4 de octubre de 2017.

26. Vargas Rodríguez AR. Repercusión del desarrollo de la biotecnología para la salud pública en Cuba. Rev Hum Med. 2014;14(1):206-19. Disponible en: http:// scielo.sld.cu/pdf/hmc/v14n1/hmc13114. pdf Acceso el 21 de abril de 2017.

27. Sotolongo Padrón F, Campa Huergo C, Casanueva Gil V, Fajardo Díaz EM, Cuevas Valdespino IE, González Gotera N. Cuban meningococcal $\mathrm{BC}$ vaccine: experiences and contributions from 20 years of application. MEDICC Rev. 2009;9(1):16-22. Disponible en: http://www.medicc.org/ mediccreview $/$ index. php? issue $=6 \&$ id $=$ $61 \& a=v a h t m l$ Acceso el 24 de septiembre de 2017.

28. Mendoza Hernández I, Cachimaille Benavides Y, Guerra Chaviano PP, Robaina García M, Dámaso Fernández J, Wilford de León $\mathrm{M}$, et al. Impacto en la asistencia médica cubana de la extensión nacional del ior®LeukoCIM a través de ensayos clínicos. Rev Cubana Farm. 2010;44(supl 2):1-14. Disponible en: http://bvs.sld.cu/ revistas/far/vol44_sup2_10/Impacto $\% 20$ en $\% 201$ \% $\% 20$ asistencia $\% 20$ medica $\% 20$ cubana $\% 20 \mathrm{de} \% 201 \mathrm{a} \% 20$ extension $\% 20$ nacional\%20del\%20ior\%20LeukoCIM\%20a\%20 traves\%20de\%20EC.pdf Acceso el 24 de septiembre de 2017.

29. Saavedra D, Crombet T. CIMAvax-EGF: a new therapeutic vaccine for advanced non-small cell lung cancer patients. Front Immunol. 2017;8:269. Disponible en: https: / / www.ncbi.nlm.nih.gov/pmc/articles / PMC5346887/ Acceso el 3 de octubre de 2017.

30. Berlanga J, Fernández JI, López E, López PA, del Río A, Valenzuela C, et al. Heberprot-P: a novel product for treating advanced diabetic foot ulcer. MEDICC Rev. 2013;15(1):11-5. Disponible en: http://www.medicc.org/ mediccreview $/$ index.php issue $=23 \&$ id $=287 \& \mathrm{a}=\mathrm{vahtml}$ Acceso el 2 de octubre de 2017.

31. García R, Suárez R. Resultados de la estrategia cubana de educación en diabetes tras 25 años de experiencia. Rev Cubana Salud Publica. 2007;33(2):1-16. Disponible en: http:/ / scielo.sld.cu/pdf/rcsp/v33n2/ spu08207.pdf Acceso el 19 de abril de 2017.

32. García R, Suárez R. La educación a personas con diabetes mellitus en la atención primaria de salud. Rev Cubana Endocrinol. 2007;18(1). Disponible en: http:/ / scielo.sld. $\mathrm{cu} /$ scielo.php? script $=$ sci_arttext \& pid=S1561-29532007000100005 Acceso el 19 de abril de 2017.

33. República de Cuba, Ministerio de Salud Pública. Guías para la asistencia integral a 
pacientes con dengue. La Habana: Editorial Ciencias Médicas; 2012. Disponible en: http:/ / www.cimgbc.sld.cu/Articulos / guia_asist_pacient_dengue_completo.pdf Acceso el 19 de abril de 2017.

34. Organización Panamericana de la Salud. Dengue y dengue hemorrágico en las Américas: guías para su prevención y control. Washington, DC: OPS; 1995. (Publicación Científica, 548). Disponible en: http:// www1.paho.org/Spanish/HCP/HCT/ VBD/arias-dengue.htm Acceso el 19 de abril de 2017.

35. World Health Organization. Dengue guidelines for diagnostic, treatment, prevention and control. Geneva: WHO; 2009. Disponible en: http://www.who.int/tdr/ publications/documents/dengue-diagnosis.pdf Acceso el 21 de abril de 2017.

36. Barniol J, Gazkowski R, Vega Barbato E, Benancio Danhuna R, Salgado TM, Martínez $\mathrm{E}$, et al. Usefulness and applicability of the revised dengue case classification by disease: multi-centre study in 18 countries. BMC Infect Dis. 2011;11:106. Disponible en: http:/ / bmcinfectdis.biomedcentral.com/ articles/10.1186/1471-2334-11-106 Acceso el 21 de abril de 2017.

37. Colectivo de Autores. Por la vida. La Habana: Casa Editora Abril; 2004.

38. Añé Aguiloche L, Granda Dihigo J. La asistencia social en Cuba. Su situación actual y principales programas. La Habana: Centro de Estudios de Población y Desarrollo; 2008. Disponible en: http:// www.one.cu/publicaciones/cepde/cuaderno/Articulo\%206.pdf Acceso el 2 de octubre de 2017.

39. González Fernández RS, González Fernández A. Cervical cancer control: potential benefits from intersectoral action between biotechnology and public health. MEDICC Rev. 2016;18:54-7. Disponible en: http://www.medicc.org/mediccreview $/$ index.php?issue $=38 \& i d=521 \& a=v-$ ahtml Acceso el 2 de octubre de 2017.

40. Luis-Gonzálvez IP. Personal responsibility in Cuba's universal health model. MEDICC Rev. 2017;19(2-3):76. Disponible en: http://www.medicc.org/mediccreview $/$ index.php issue $=43 \& i d=595 \& a=v-$ ahtml Acceso el 2 de octubre de 2017.
41. Guzmán MG, Álvarez A, Fuentes O, Kourí G. Enfermedades de la pobreza, el caso del dengue. An Acad Ciencias Cuba. 2012;2(1). Disponible en: http://www.revistaccuba. $\mathrm{cu} /$ index.php/acc/article/viewFile/109/91 Acceso el 2 de octubre de 2013.

42. Rojo Pérez N, Castell-Florit Serrate P Torres Rojo R. Políticas sanitarias y gestión social para el control del dengue en Cuba. La Habana: Editorial Lazo Adentro; 2013.

43. Portuondo M, Cobas M, Zacca E, López J, Lantigua A, Boligán Y, et al. Improving disability care in countries of the Bolivarian Alliance for the Peoples of Our America. MEDICC Rev. 2016;18(3). Disponible en: http:/ / www.medicc.org/mediccreview / index.php?issue $=40 \& i d=549 \& a=$ vahtml Acceso el 20 de abril de 2017.

Manuscrito recibido el 28 de abril de 2017. Aceptado para publicación, tras revisión, el 20 de octubre de 2017.

ABSTRACT In Cuba, health research is based on the priorities of national scientific policy, derived from the health status of the population. The objective of this article is to describe the characteristics of the System of Science and Technological Innovation and how the

\section{Science and technological innovation in health in Cuba: results in selected problems}

Keywords results of its research benefit the health of the population groups. To this end, research related to the generation of products and technologies, diabetes, dengue and disability was selected. This system follows a methodology outlined by the Ministry of Science, Technology and Environment and has 37 research entities. It is organized into programs and projects that favor basic and applied research, with a multidisciplinary and intersectoral approach; these programs and projects are funded mostly by the State and are organized in self-contained cycles, i.e., the same entity is responsible for the entire process, from research to marketing, including market studies and post-marketing surveillance. The selected research shows an alignment between the research, the generalization of the results and its effect in improving health and universal access to health in the population. Positive results were obtained in diagnostic methods, preventive and therapeutic vaccines, warning signs for the prognosis and treatment of dengue, prevention of congenital malformations, and policies and programs that have benefited people with disabilities and their families. The will of the State to develop and fund scientific research, intersectoral action, the definition of research priorities, and the systematic training and attention to human resources have been key factors for the fulfillment of the objectives of the system.

National science, technology and innovation policy; health sciences, technology and innovation management; biomedical research; health programs and plans; research design; Cuba. 
RESUMO Em Cuba, a pesquisa em saúde baseia-se nas prioridades da política científica nacional, derivadas do estado de saúde da população. O objetivo deste artigo é descrever as características do Sistema de Ciência e Inovação Tecnológica e como os resultados de

Ciência e inovação tecnológica em saúde em Cuba: resultados em problemas selecionados

Palavras-chave suas pesquisas beneficiam a saúde dos grupos populacionais. Para este fim, foram selecionadas pesquisas relacionadas à geração de produtos e tecnologias, diabetes, dengue e deficiência. Este sistema segue a metodologia do Ministério da Ciência, Tecnologia e Meio Ambiente e possui 37 entidades de pesquisa. Está organizado em programas e projetos que favorecem pesquisas básicas e aplicadas, com abordagem multidisciplinar e intersetorial; estes são financiados principalmente pelo Estado e organizados em ciclos fechados ou completos, ou seja, a mesma entidade é responsável por todo o processo, desde pesquisa até marketing, incluindo estudos de mercado e vigilância pós-comercialização. As pesquisas selecionadas mostram a harmonia entre a pesquisa, a generalização dos resultados e seus efeitos na melhoria da saúde e no acesso universal à saúde na população. Resultados positivos foram obtidos em métodos de diagnóstico, vacinas preventivas e terapêuticas, sinais de alerta para o prognóstico e tratamento da dengue, prevenção de malformações congênitas e políticas e programas que beneficiaram pessoas com deficiência e suas famílias. A vontade do Estado de desenvolver e financiar pesquisas científicas, ações intersetoriais, a definição de prioridades de pesquisa e o treinamento e atenção sistemática do capital humano têm sido fatores determinantes para o cumprimento dos objetivos do sistema.

Política nacional de ciência, tecnologia e inovação; gestão de ciência, tecnologia e inovação em saúde; pesquisa biomédica; planos e programas de saúde; projetos de pesquisa; Cuba. 